\title{
The effects of interpersonal affect on errors made when reconstructing a stimulus display
}

\author{
ROBERT KLECK \\ DARTMOUTH COLLEGE
}

Replacement distance errors made while reconstructing a stimulus display were examined as a function of the affective relationship existing between the stimuli. As expected photographs of persons highly liked were placed closer to a photo of self than were photographs of persons not highly liked. Stimulus pairs of highly liked persons were also placed closer than pairs involving one person high and one low in liking. There was some suggestion that female stimuli result in more proximate placement than male stimuli.

The present experiment is one of a series (Kleck \& Nuessle, in press) investigating nonverbal cues which may function as either indicators or communicators of interpersonal affect. A number of recent studies (e.g., Little, 1965; Levinger \& Gunner, 1967) have suggested that when persons are asked to structure representations of interactions between persons these constructions will be influenced by the degree of positive affect attributed to the interaction.

If placement of the highly stylized forms used in these studies is sensitive to the affective relationship ascribed to the forms, it is possible that the affective relationship existing between two stimulus objects, independent of experimenter ascriptions, can have a similar effect. If this should prove to be true, such behavior may serve as a nonverbal index to the affective attraction which a person associates with an interaction involving specific persons. The study reported below is an attempt to examine this possibility.

The approach employed in the present study is a modification of the Kuethe Felt Figure technique (Kuethe, 1962). As in Kuethe, the $S$ views the placement of two rectangles on a large felt board, then, with the rectangles removed, he attempts to place two stimuli where the rectangles have been. The dependent measure is the error that is made in replacement. In the present study the stimuli are enlarged photographs of real persons. These persons differ in the degree of their acquaintanceship with the $S$ and in the interpersonal attraction which they hold for him. The general proposition to be tested is that greater errors of replacement or reconstruction will be made for stimuli involving a positive affective relationship than for stimuli involving no or a negative relationship. These errors will be in the direction of placing the stimuli too close relative to the stimulus display being reconstructed. The design also permitted us to explore replacement errors as a function of sex of stimulus object. While the study is frankly exploratory, given the manner in which the stimuli differ from those of previous studies, the following hypotheses were formulated as reasonable expectations: (a) A picture of self paired with a picture of a close friend will result in closer placement than will a pair of stimuli composed of self and a disliked person; (b) Pairs of stimuli, both of which are highly liked, will be placed more proximate than will pairs of stimuli in which one is highly liked and one not; (c) Pairs of photographs of unknown females will be placed closer than pairs of photographs of unknown males.

Hypothesis (b) in part assumes the balance notion that highly liked peers will be seen to also like each other. The last hypothesis derives from Kuethe's (1962) finding that representative female figures are typically placed closer than representative male figures. Subjects

The Ss for this experiment were 20 male college undergraduates who had just completed a course in human relations in which they were members of small (7-10 persons) face-to-face groups for one college term. Procedure

Two months prior to the experiment proper, two right and left profile photographs were taken of each $S$ and enlarged such that the scale of the final photograph was approximately $1 \mathrm{in}$. to $1 \mathrm{ft}$. The background was trimmed from the photographic enlargements and each stimulus was backed with felt. Similar stimuli were constructed from the photographs of four females who were age peers of the Ss but unknown to them. These stimuli were such that they readily adhered to a vertical felt board, and had the advantageous characteristic of leaving no mark on the field which could be used as a cue for subsequent placements.

Following Kuethe, a large piece of blue felt ( $6 \times 8 \mathrm{ft}$ ) was stretched against one wall of the experimental room. On each trial two rectangles were placed 26 in. apart in the central region of the stimulus field. The $\mathrm{S}$ was permitted to view the stimulus display from a distance of $12 \mathrm{ft}$ for $5 \mathrm{sec}$. The $E$ then removed the rectangles and asked $S$ to reconstruct the stimulus display by placing the flannel backed photograph where the rectangles had been on the large felt background. The instructions to $\mathrm{S}$ prior to the first trial emphasized that this was a task in "visual memory" in which the central experimental question "concerns a person's ability to accurately reconstruct what he sees." A period of 15 sec elapsed between placement trials and 
Table 1. Average placement distances between stimulus pairs

\begin{tabular}{lcc} 
Stimulus Pairs & Mean distance in in. & SD \\
\hline Self-Unknown female & 20.94 & 3.79 \\
Liked male-liked male & 21.70 & 3.51 \\
Self-self & 21.80 & 3.59 \\
Self-most liked male & 22.02 & 2.74 \\
Unknown female-unknown female & 22.07 & 3.44 \\
Self-unknown male & 22.66 & 3.26 \\
Unknown male-unknown male & 22.68 & 3.84 \\
Self-least liked male & 23.12 & 2.79 \\
Most-least liked male & 23.27 & 3.83 \\
\hline
\end{tabular}

$\mathrm{S}$ was asked to look at the stimuli for the next placement during the interval. This procedure was used to focus S's attention on the nature of each stimulus pair prior to placement. To further draw S's attention to the photographs, E named the persons in the photos as he handed them to $\mathrm{S}$. Each pair was composed of one right and one left profile such that when placed side by side, they appeared to be looking at each other. Two "warmup" trials were run using geometric forms and if $\mathrm{S}$ had any questions or indicated by his behavior that he did not understand the procedure, it was clarified for him.

The stimulus pairs employed for each $\mathrm{S}$ included the pairs listed in Table 1. The order in which $S$ was given these stimulus pairs was randomized for each $S$. Liking was determined on the basis of a sociometric questionnaire given in such a way as to dissociate it from the present experiment. The relevant question on the sociometric device asked $S$ to place all persons in his small group on a 100 point liking scale such that no two persons fell at the same point. The top and bottom persons were taken as "most" and "least" liked stimuli. The $E$ conducting the experiment was not informed regarding which were the positive and which the negative affect pairs.

At the conclusion of the study $S$ was interviewed concerning his reactions to the experiment. After all Ss had participated in the experiment, the design and results were described to them.

\section{Resulis and Discussion}

The first question of interest is how the Ss perceived the experiment and the purpose which they ascribed to it. The primary response to the postexperimental interview reiterated the $E$ 's experimental instructions, i.e., that $E$ was interested in accuracy of placement of the stimuli. Two Ss indicated that liking may have had something to do with E's interests in that liking may be expected to improve the accuracy of placement (a hypothesis just counter to that actually being tested). Only one $S$ verbalized the notion that liking would tend to reduce the distance between the two photographs when placed on the felt board and his data were excluded from the analysis.

The results for all sets of stimulus pairs are presented in Table 1, and are rank ordered with regard to size of replacement error. The replacement error measurement was done to the nearest $1 / 16$ in., and was based on the nose to nose distance between stimuli. The comparison relevant to the first hypothesis is the difference between self-most liked and self-least liked pairs. As can be seen from Table 1 this difference is in the predicted direction in that the former are placed more proximately. This difference is statistically significant $(t=2.148, \mathrm{df}=18, \mathrm{p}<.05)$.

The means relevant to the second hypothesis are those for high liked-high liked and high liked-low liked stimulus pairs. These pairs were constructed for the former by using the persons ranked highest and next highest on the liking scale and for the latter by pairing persons ranked highest and lowest on the same scale. Again the differences are in the expected direction and again they are significant $(t=2.988, d f=18, p<.01)$.

The hypothesis regarding the influence of sex of stimulus figure on replacement error is not supported as can be seen by comparing the female-female placement distances with the male-male placement distances $(t=1.33, \mathrm{~ns})$, though the differences are in the expected direction. It is interesting, however, to examine what happens when a photo of self is paired with either an unknown female or an unknown male. As the means in Table 1 indicate, greater errors in the direction of close placement are made in the former $(t=4.127, \mathrm{df}=18, \mathrm{p}<.001)$. On a post hoc basis this might be viewed as partial support for the predicted sex effect.

The results support the notion that affective attraction introduces consistent errors during the process of reconstructing a stimulus display using photographs of real persons. The task may, therefore, prove to be of some value as a nonverbal assessment technique in situations where there is some reason to doubt the validity of self report (e.g., Kleck, in press).

\section{References}

KLECK, R. Physical stigma and nonverbal cues emitted in face-to-face interaction. Hum. Relat., in press, 1968.

KLECK, R. \& NUESSLE, W. Congruence between indicative and communicative functions of eye contact in interpersonal relations. Brit. $J$. soc. clin. Psychol., in press, 1968.

KUETHE, J. L. Social Schemas. J. abnorm. soc. Psychol., 1962, 64, 31-38.

LEVINGER, G. \& GUNNER, J. The interpersonal grid: I. Felt and tape techniques for the measurement of social relationships. Psychon. Sci., 1967, 8, 173-174.

LITTLE, K. B. Personal Space. J. exp. soc. Psychol., 1965, 1, 237-247. 